\title{
Automatic Segmentation and Labelling of Multi-Lingual Speech Data
}

\author{
A. Vorstermans ${ }^{\dagger}$, J.P. Martens ${ }^{\circ *}$ and B. Van Coile ${ }^{\dagger}$ \\ ${ }^{\circ}$ ELIS, University of Gent, St.-Pietersnieuwstraat 41, B-9000 Gent, Belgium \\ $\dagger$ Lernout \& Hauspie Speech Products NV, Sint Krispijnstraat 7, B-8900 Ieper, Belgium
}

Mailing address:

Annemie Vorstermans

ELIS

University of Gent

St.-Pietersnieuwstraat 41

B-9000 Gent

Belgium

${ }^{*}$ Senior Research Associate of the National Fund for Scientific Research 
Number of pages: 48

Number of tables: 5

Number of figures: 10

Running headline: Segmentation and Labelling

Keywords: automatic segmentation and labelling, multi-lingual, neural networks 


\section{List of Tables}

1 The performance of the automatic segmentation and labelling system on the training and test set of the Flemish data (using auditory phonemic

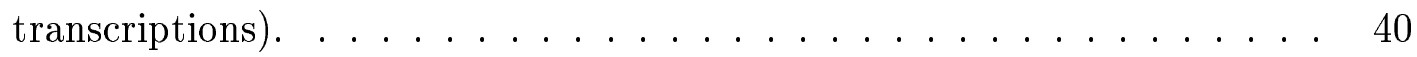

2 The performance of the automatic segmentation and labelling system on Dutch, French, American English, Spanish and Korean isolated word databases. . . . . . . . . . . . . . . . . . . . . . . . . . 44 4

3 Error percentages of the automatic segmentation and labelling system (baseline and adapted) for the core test of the TIMIT continuous speech database. . . . . . . . . . . . . . . . . . . .

4 Error percentages of the automatic annotation system trained using the manual labellings, for the core test of TIMIT. . . . . . . . . . . .

5 Mean error percentages of the automatic labelling system for the English, Danish and Italian portions of EUROM0 (baseline system, adaptation using 3 speakers and adaptation to 1 speaker). . . . . . . . . . 


\section{List of Figures}

1 An example of the state-transition model that represents the transcription of the Flemish sentence de tijd. . . . . . . . . . . . . . . 35

2 Block-diagram of the automatic segmentation and labelling system. . . . 36

$3 \quad$ Phoneme models with (a) one, (b) two or (c) three emitting transitions. . 37

4 Reduction of the effect of fluctuations in $E(n) \ldots \ldots . \ldots$

5 Percentage of discriminative boundaries, percentage of correctly classifiable phonetic segments and global accuracy as a function of the number of phonetic classes. . . . . . . . . . . . . . 39

6 Some examples of the major deletion errors encountered in the isolated word corpora. . . . . . . . . . . . . . .

7 Some examples of automatically placed boundaries that cannot be considered as being misplaced. . . . . . . . . . . . . . 46

8 Some differences between the automatic and the manual segmentation and labelling of data. The pictures show that some of the deletions, $h h$ (TIMIT) and $D$ (English EUROM0), should not be counted as deletions.

9 Some systematic differences between the automatic and the manual segmentation and labelling of data. The top figure shows a deletion due to the unclear transition between the phones $w$ and $e$ (English EUROM0). The bottom figure is an example of a segmentation and labelling error that is due to the discrepancy between the standard phonetic transcription used at the input of our system, and the manual label string provided with the data $($ TIMIT) $\ldots \ldots \ldots \ldots$

10 Some examples of manual segmentation errors (top: w, TIMIT; bottom:

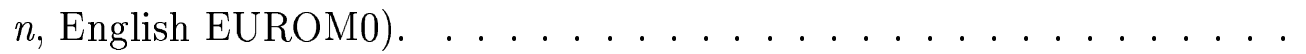


Abstract. A new system for the automatic segmentation and labelling of speech is presented. The system is capable of labelling speech originating from different languages without requiring extensive linguistic knowledge or large (manually segmented and labeled) training databases of that language. The system comprises small neural networks for the segmentation and the broad phonetic classification of the speech. These networks were originally trained on one task (Flemish continuous speech), and are automatically adapted to a new task. Due to the limited size of the neural networks, the segmentation and labelling strategy requires but a limited amount of computations, and the adaptation to a new task can be accomplished very quickly. The system was first evaluated on five isolated word corpora designed for the development of Dutch, French, American English, Spanish and Korean text-to-speech systems. The results show that the accuracy of the obtained automatic segmentation and labelling is comparable to that of human experts. In order to provide segmentation and labelling results which can be compared to data reported in the literature, additional tests were run on TIMIT and on the English, Danish and Italian portions of the EUROM0 continuous speech utterances. The performance of our system appears to compare favourably to that of other systems.

Résumé. Dans cet article, un nouveau système pour la segmentation et l'étiquetage automatique de la parole est presenté. Notre système est capable de transcrire des paroles d'origines diverses sans demander une connaissance linguistique extensive ou une base de données démesurée (segmentée et étiquetée manuellement). Le système contient des petits réseaux neuronnaux pour la segmentation et la classification phonétique globale, qui ont d'abord été entrainés en vue d'une tache (la parole continue en Flamand) et qui sont automatiquement adaptés à de nouvelles taches. A cause de la taille limitée des réseaux neuronnaux, la segmentation et l'étiquetage requièrent bien évidemment une limitation des temps de calculs et une capacité d'adaptation rapide vers des nouvelles taches. Le système est evalué en utilisant cinq corpus de mots isolés, ébauchés pour le développement des systèmes TTS néerlandais, français, anglais-américain, espagnol et coréen. Les résultats montrent que la précision de notre système est comparable à celle d'experts humains. Pour obtenir des résultats concernant la segmentation et l'étiquetage, comparable avec des résultats publiés dans la littérature, des tests additionnels ont été effectués sur TIMIT et les parties anglaise, danoise et italienne de EUROM0. La performance de notre système se compare favorablement avec celles des autres systèmes automatiques.

Zusammenfassung. In diesem Artikel wird ein neues System der automatischen Segmentierung und Etikettierung von Sprache vorgestellt. Das System ist imstande die meis- 
ten Sprachen zu etikettieren ohne daß eine umfassende linguistische Kenntnis oder eine große (manuell segmentierte und etikettierte) Datenbank erforderlich ist. Das System enthält kleine neurale Netzwerke für die Segmentierung und die rohe phonetische Klassifizierung, die ursprünglich nur in einer Domäne ausgeführt wurde (in flämisch) und sich dann automatisch an eine neue Aufgabenstellung anpaßt. Auf Grund der Begrenztheit des neuralen Netzwerkes nehmen die Segmentierungs- und Etikettierungsstrategien nur ein beschränktes Berechnungskontingent in Anspruch und außerdem ist die Anpassung an eine neue Aufgabe sehr schnell bewerkstelligbar. Das System wurde anhand von fünf isolierten Wortkörpern, die für die Entwicklung von Text-Sprache-Umsetzung in Niederländisch, Französisch, amerikanischem Englisch, Spanisch und Koreanisch entworfen wurde, ausgewertet. Die Ergebnisse zeigen, daß die Genauigkeit unseres automatischen Segmentierungs- und Etikettierungssystem mit dieser von menschlichen Experten gleich zu stellen ist. Um unsere Segmentierungs- und Etikettierungsergebnisse mit ähnlichen in der Literatur angegebenen Resultaten vergleichbar zu machen, wurden beikommende Tests auf der TIMIT und auf den englischen, dänischen und italienischen Partitionen der EUROM0 für fließende Sprachäußerungen, durchgeführt. Ein Leistungsvergleich verschiedener Systeme fällt zu Gunsten des unsrigen aus. 


\section{Introduction}

In speech research and development, there is a great need for segmented and labelled (i.e. annotated) databases. To develop a speech synthesis system based on segment concatenation, one needs a large, segmented and labelled (single-speaker) database of isolated word utterances from which the diphones and/or larger segments such as triphones of the segment inventory can be extracted. In addition, an annotated continuous speech database is required in order to learn the prosody in fluent speech. Segmented and labelled speech data is also needed for the development and evaluation of speech recognition systems and for corpus-based phonetic research.

Manual segmentation and labelling however exhibits some major drawbacks. For one, the manual intervention in this process is extremely time consuming and tedious. Moreover, as mentioned by Cosi et. al. [Cosi et al 1991], Torkolla [Torkkola 1988] and Van Erp [van Erp \& Boves 1988], it is almost impossible to reproduce the manual segmentation results, due to the variability of human visual and acoustical perceptual capabilities, and to the difficulty of establishing a clear common labelling strategy. Consequently, the manual segmentation and labelling procedure is implicitly inconsistent. Furthermore, it requires expert skills.

An automatic system is not faultless but it is, inherently, consistent and the results are reproducible. Ideally, one would like to have an automatic labelling system which is capable of handling isolated and continuous speech, multiple speakers and multiple languages. As such a system may be unreachable with today's speech technology, we have chosen to develop a system that is not entirely task-independent, but at least retrainable in an unsupervised way, i.e. without requiring any manual segmentation and labelling, whenever new data (new speaker, language or speech mode) is submitted to labelling.

In the literature, many automatic labelling systems have been reported (a.o. [Arai et al 1990, Brugnara et al 1993, Leung \& Zue 1984, Ljolje \& Riley 1991, Torkkola 1988, van Hemert 1985, Wang et al 1990]) but only a few of them were evaluated on different tasks. Svendsen et al. [Svendsen \& Kvale 1990] performed independent tests on English and Italian. Kabré et al. [Kabré et al 1991] report results on the EUROM0 English, French and Swedish databases, but with a system requiring a supervised language dependent training. Kvale [Kvale 1993] conceived a constrained HMM system 
and obtained more than $80 \%$ correctly placed phoneme boundaries (within $20 \mathrm{~ms}$ ) for the EUROM0 data (again after a language dependent supervised training). Only Dalsgaard et al. [Dalsgaard 1992, Dalsgaard et al 1991b] report results on English and Italian obtained with a system which was trained on Danish data and which performs a two-stage phoneme label alignment. In the first stage, a self-organizing neural network transforms the signal parameters into a set of continuously valued acoustic-phonetic features. In the second stage, the label alignment is performed according to a Viterbi decoding/level building technique. Using this strategy on continuous speech (EUROM0), 60.5\% (for English) to $47 \%$ (for Italian) of the segment boundaries are positioned within $20 \mathrm{~ms}$ of the corresponding manually placed boundaries.

In this paper, a new automatic segmentation and labelling system is described. It is the result of a new mathematical deduction of the most likely segmentation and labelling, given the acoustic observations and the transcription of the utterance. By introducing a factorisation of the probabilities involved, a modular system could be conceived. By introducing intermediate levels of segmentation and phonetic classification in the acoustic modelling, an easy portability to other languages could be attained: some of the modules turn out to perform equally well across different languages.

The new system was evaluated on Dutch, French, American English, Spanish and Korean single-speaker isolated word databases, on TIMIT, an American English multispeaker continuous speech database [Lamel et al 1986] and on the English, Danish and Italian continuous speech passages of the EUROM0 database [Grice et al 1989].

\section{The Lexical Input}

Although the segmentation and labelling of the speech data is performed at the acousticphonetic level ([Barry \& Fourcin 1992]), the transcription of an utterance that is submitted to our system can be one of the following levels ([Barry \& Fourcin 1992, Kvale 1993]):

1. a citation, canonical or standard phonemic transcription;

2. a phonotypical or text-to-phoneme phonemic transcription;

3. an auditory phonemic transcription; 
4. an audio-visual or manual-labelling phonetic transcription.

The first transcription represents the concatenation of the standard pronunciations of the words contained in the uttered sentence. No assimilations nor coarticulations are taken into account. If the context of the words is taken into account, a phonotypical or text-to-phoneme transcription is obtained. This transcription represents a possible pronunciation of the sentence as a whole. The auditory transcription on the other hand is obtained by listening carefully to the speech signal. It consists of the phonemes which were actually perceived by the listener. These three kinds of transcriptions are all phonemic transcriptions, i.e. sequences of phonemes. The last transcription however is a sequence of acoustically motivated sound segments, called phonetic units or phones (as the ones provided with the TIMIT database). These phones have relatively stable or constantly changing acoustic-phonetic properties, and can be identified by an audiovisual inspection of the speech signal and/or its spectral representation.

The following is an example of the different transcriptions that will be used in the reported experiments and that may arise from a particular orthographical representation. The example is extracted from the TIMIT database.

orthographical: romantic gift

citation: $\quad \quad \quad \quad$ ow $\mathrm{m}$ ae $\mathrm{n} \mathrm{t}$ ih $\mathrm{kg}$ ih $\mathrm{f} \mathrm{t}$

phonotypical: $\quad \mathrm{r}$ ow $\mathrm{m}$ ae $\mathrm{n} \mathrm{t}$ ih $\mathrm{g}$ ih $\mathrm{ft}$

auditory: $\quad \mathrm{r}$ ax $\mathrm{m}$ ae $\mathrm{nx}$ ix $\mathrm{kg}$ ih $\mathrm{f} \mathrm{t}$

manual-labelling: $\mathrm{r}$ ax $\mathrm{m}$ ae $\mathrm{nx}$ ix $\mathrm{kcl} \mathrm{g}$ ih $\mathrm{f} \mathrm{tcl} \mathrm{t}$

As the segmentation and labelling in our system will be performed in terms of phones, the citation, the phonotypical and the auditory phonemic transcriptions have to be translated into phone sequences. This translation (see section 4.1) will emerge in a state-transition model as the one depicted on figure 1. This model consists of phone and Figure 1 deletion arcs, and implies context-independent phones. All deletion arcs are indicated by the same symbol $\phi$, but the transition probabilities of these arcs can be different for different phones. The deletion arcs are required to leave open the possibility that a phone emerging from the transcription was not pronounced. 


\section{Formal Description of the Annotation Problem}

Before starting the formal description of the annotation problem, it is useful to introduce some definitions and symbols:

- The phonetic segmentation of the utterance is the sequence of consecutive time intervals coinciding with the subsequent phones. The boundaries between these phones are called phonetic boundaries.

- $\vec{s}$ : a candidate phonetic segmentation of the utterance. The sequence $\left(s_{0}, s_{1}, \cdots, s_{I}\right)$ consists of the candidate phonetic segment boundaries delimiting the subsequent phones. The boundaries $s_{0}$ and $s_{I}$ point to the beginning and the end of the utterance respectively.

- $\vec{u}$ : a possible sequence of phones $\left(u_{1}, \cdots, u_{I}\right)$ realised in the candidate phonetic segments $\left(s_{i-1}, s_{i}\right)$ with $(i=1, \cdots, I)$.

- $\vec{c}$ : a sequence of phonetic properties or classes which can be attached to the phonetic segments of $\vec{s}$. These classes represent an intermediate level of phonetic detail to be derived from the acoustic observations in the segments of $\vec{s}$.

- $\vec{t}$ : the state-transition model that was derived from the transcription provided with the utterance.

- $\overrightarrow{t_{k}}$ : the state-transition model corresponding to some particular utterance that can occur within the task. This model is supposed to have $L_{k}$ transitions (and $L_{k}+1$ states).

- $\vec{o}$ : the sequence of acoustic observation vectors extracted by the acoustic front-end. Every $10 \mathrm{~ms}$ a vector $\overrightarrow{o_{n}}(n=1, \cdots, N)$ is generated.

- $\vec{b}$ : a set of candidate phonetic boundaries. These boundaries can coincide with the frame boundaries (every $10 \mathrm{~ms}$ ) or they can represent an intermediate level of segmentation emerging from an initial segmentation algorithm.

- $\lambda_{A}$ : the set of acoustic models (contains information about the acoustic properties of the phones expressed in terms of the acoustic observations). 
- $\lambda_{L}$ : the language model describes how the phonemes can be realised as a sequence of phones, and implies the transcriptions that can occur within the envisaged task.

The purpose of the automatic annotation system is to find the most likely phonetic segmentation $\hat{\vec{s}}$ and phone sequence $\hat{\vec{u}}$ of an utterance given the provided transcription and the acoustic observations for that utterance:

$$
(\hat{\vec{s}}, \hat{\vec{u}})=\arg \max _{(\vec{s}, \vec{u})} P(\vec{s}, \vec{u} \mid \vec{o}, \vec{b}, \vec{t})
$$

In order to estimate the probability $P(\vec{s}, \vec{u} \mid \vec{o}, \vec{b}, \vec{t})$ that $(\vec{s}, \vec{u})$ is the correct annotation given the evidence, we use a set of acoustic models $\lambda_{A}$, derived from a 'large' number of utterances which are supposed to be representative for the task. These utterances imply a language model $\lambda_{L}$. We will thus calculate the probability $P\left(\vec{s}, \vec{u} \mid \vec{o}, \vec{b}, \vec{t} ; \lambda_{L}, \lambda_{A}\right)$. It can be rewritten as:

$$
P\left(\vec{s}, \vec{u} \mid \vec{o}, \vec{b}, \vec{t} ; \lambda_{L}, \lambda_{A}\right)=\frac{P\left(\vec{s}, \vec{u}, \vec{t} \mid \vec{o}, \vec{b} ; \lambda_{L}, \lambda_{A}\right)}{P\left(\vec{t} \mid \vec{o}, \vec{b} ; \lambda_{L}, \lambda_{A}\right)}
$$

The probability $P\left(\vec{t} \mid \vec{o}, \vec{b} ; \lambda_{L}, \lambda_{A}\right)$ is independent of $\vec{s}$ and $\vec{u}$, and is therefore irrelevant for the maximizing process. If $\vec{c}$ is a sequence of disjunct phonetic classes (which will be defined later) that can be attributed to the subsequent segments of $\vec{s}$ on the basis of the acoustic models $\lambda_{A}$, the probability in the numerator can be rewritten as:

$$
\begin{aligned}
P & =P\left(\vec{s}, \vec{u}, \vec{t} \mid \vec{o}, \vec{b} ; \lambda_{L}, \lambda_{A}\right)=\sum_{\vec{c}} P\left(\vec{s}, \vec{c}, \vec{u}, \vec{t} \mid \vec{o}, \vec{b} ; \lambda_{L}, \lambda_{A}\right) \\
& =\sum_{\vec{c}} P\left(\vec{s}, \vec{c} \mid \vec{o}, \vec{b} ; \lambda_{L}, \lambda_{A}\right) P\left(\vec{u}, \vec{t} \mid \vec{s}, \vec{c}, \vec{o}, \vec{b} ; \lambda_{L}, \lambda_{A}\right)
\end{aligned}
$$

The sum is taken over all possible sequences of phonetic properties that might be attached to the subsequent segments of $\vec{s}$. As these properties are disjunct, only one $\vec{c}$ can be the correct intermediate description of the utterance.

It is assumed that all the information in $\left(\vec{o}, \vec{b}, \vec{s} ; \lambda_{A}\right)$ which is relevant to the labelling of the utterance (i.e. relevant for determining $(\vec{u}, \vec{t})$ ), will be encoded in $\vec{c}$. Consequently:

$$
P=\sum_{\vec{c}} P\left(\vec{s}, \vec{c} \mid \vec{o}, \vec{b} ; \lambda_{L}, \lambda_{A}\right) P\left(\vec{u}, \vec{t} \mid \vec{c} ; \lambda_{L}\right)
$$

This assumption is only acceptable if duration modelling is included in $\lambda_{A}$ (to get rid of $\vec{s}$ ), and if the phonetic classes are sufficiently detailed to ensure that all the relevant information in $(\vec{o}, \vec{b})$ is effectively encoded. 
The probability $P\left(\vec{s}, \vec{c} \mid \vec{o}, \vec{b} ; \lambda_{L}, \lambda_{A}\right)$ is called the acoustical probability because it is mainly determined by the acoustical information. The probability $P\left(\vec{u}, \vec{t} \mid \vec{c} ; \lambda_{L}\right)$ is called the language probability because it is determined by the language model and by the phonetic characterisation of the utterance.

\section{The Acoustical Probability}

The acoustical probability $P\left(\vec{s}, \vec{c} \mid \vec{o}, \vec{b} ; \lambda_{L}, \lambda_{A}\right)$ can be split into two parts:

$$
P\left(\vec{s}, \vec{c} \mid \vec{o}, \vec{b} ; \lambda_{L}, \lambda_{A}\right)=P\left(\vec{s} \mid \vec{o}, \vec{b} ; \lambda_{L}, \lambda_{A}\right) P\left(\vec{c} \mid \vec{s}, \vec{o}, \vec{b} ; \lambda_{L}, \lambda_{A}\right)
$$

The first part represents the posterior probability that $\vec{s}$ is the correct phonetic segmentation given the acoustical evidence. According to Bayes' law, it can be expanded as:

$$
P\left(\vec{s} \mid \vec{o}, \vec{b} ; \lambda_{L}, \lambda_{A}\right)=\prod_{i=1}^{I} P\left(s_{i} \mid s_{0}, \cdots, s_{i-1}, \vec{o}, \vec{b} ; \lambda_{L}, \lambda_{A}\right)
$$

However, if we assume that the probability of $s_{i} \in \vec{b}$ being a phonetic boundary is solely dependent on the position of the previous phonetic boundary, and on a vector $\vec{v}_{s i}$ of acoustical variables retrieved from $(\vec{o}, \vec{b})$ and describing segment $\left(s_{i-1}, s_{i}\right)$ in its context (e.g. average spectra, segment duration, ...), then

$$
P\left(\vec{s} \mid \vec{o}, \vec{b} ; \lambda_{L}, \lambda_{A}\right)=\prod_{i=1}^{I} P\left(s_{i} \mid s_{i-1}, \vec{v}_{s i} ; \lambda_{A}\right)
$$

In determining the above probabilities, the information induced by the language model $\lambda_{L}$ was omitted implying that the phonetic/non-phonetic character of a segment can be established without any knowledge of the a priori probabilities of the phone sequences implied by $\lambda_{L}$.

The second part of equation (1) represents the posterior probability that $\vec{c}$ is the correct sequence of phonetic properties (classes) given the correct phonetic segmentation of the utterance. Again, using Bayes' law, this probability can be expanded to

$$
P\left(\vec{c} \mid \vec{s}, \vec{o}, \vec{b} ; \lambda_{L}, \lambda_{A}\right)=\prod_{i=1}^{I} P\left(c_{i} \mid c_{1}, \cdots, c_{i-1}, \vec{s}, \vec{o}, \vec{b} ; \lambda_{L}, \lambda_{A}\right)
$$


The information in $(\vec{s}, \vec{o}, \vec{b})$ which is relevant to the determination of $c_{i}$ can be collected in a vector $\vec{x}_{s i}$ describing segment $\left(s_{i-1}, s_{i}\right)$ in its context. Furthermore, if contextindependent models are assumed, the determination of $c_{i}$ does not dependent on the properties $c_{1}, \cdots, c_{i-1}$ of the previous segments. Consequently:

$$
P\left(\vec{c} \mid \vec{s}, \vec{o}, \vec{b} ; \lambda_{L}, \lambda_{A}\right)=\prod_{i=1}^{I} P\left(c_{i} \mid \vec{x}_{s i} ; \lambda_{L}, \lambda_{A}\right)
$$

\section{The Language Probability}

After repeatedly applying Bayes' rule, the language probability can be rewritten as:

$$
\begin{aligned}
P\left(\vec{u}, \vec{t} \mid \vec{c} ; \lambda_{L}\right) & =\frac{P\left(\vec{u}, \vec{t}, \vec{c} \mid \lambda_{L}\right)}{P\left(\vec{c} \mid \lambda_{L}\right)} \\
& =\frac{P\left(\vec{c} \mid \vec{u}, \vec{t} ; \lambda_{L}\right) P\left(\vec{u} \mid \vec{t} ; \lambda_{L}\right) P\left(\vec{t} \mid \lambda_{L}\right)}{P\left(\vec{c} \mid \lambda_{L}\right)}
\end{aligned}
$$

If the phone sequence $\vec{u}$ is known, the transcription $\vec{t}$ is no longer of importance because the emissions occurring on a transition are already supposed (by fig. 1) to be independent of the identity of the surrounding phones. Consequently, the language probability can be written as:

$$
P\left(\vec{u}, \vec{t} \mid \vec{c} ; \lambda_{L}\right)=\frac{P\left(\vec{c} \mid \vec{u} ; \lambda_{L}\right) P\left(\vec{u} \mid \vec{t} ; \lambda_{L}\right) P\left(\vec{t} \mid \lambda_{L}\right)}{P\left(\vec{c} \mid \lambda_{L}\right)}
$$

The probability $P\left(\vec{c} \mid \lambda_{L}\right)$ is equal to $\sum_{k} P\left(\vec{c}, \overrightarrow{t_{k}} \mid \lambda_{L}\right)$ with the sum taken over all possible transcriptions in $\lambda_{L}$. A further assumption that we make is that $\vec{c}$ is detailed enough to guarantee that only one transcription in $\lambda_{L}$ can, with a high probability, emit the sequence $\vec{c}$. In that case, the sum can be replaced by a maximum and the probability can be approximated by $\max _{k} P\left(\vec{c} \mid \overrightarrow{t_{k}} ; \lambda_{L}\right) P\left(\overrightarrow{t_{k}} \mid \lambda_{L}\right)$. If we further assume that all transcriptions are equally likely $\left(P\left(\overrightarrow{t_{k}} \mid \lambda_{L}\right)=P\left(\vec{t} \mid \lambda_{L}\right)\right)$, then we can derive that:

$$
P\left(\vec{u}, \vec{t} \mid \vec{c} ; \lambda_{L}\right) \approx \frac{P\left(\vec{c} \mid \vec{u} ; \lambda_{L}\right) P\left(\vec{u} \mid \vec{t} ; \lambda_{L}\right)}{\max _{k} P\left(\vec{c} \mid \overrightarrow{t_{k}} ; \lambda_{L}\right)}
$$

As we have introduced state-transition models with context-independent phone arcs, $P\left(\vec{c} \mid \vec{u} ; \lambda_{L}\right)$ is approximated by a product of emission probabilities:

$$
P\left(\vec{c} \mid \vec{u} ; \lambda_{L}\right)=\prod_{i=1}^{I} P\left(c_{i} \mid u_{i} ; \lambda_{L}\right)
$$


Likewise, $P\left(\vec{u} \mid \vec{t} ; \lambda_{L}\right)$ is given by

$$
P\left(\vec{u} \mid \vec{t} ; \lambda_{L}\right)=\prod_{i=1}^{I} P\left(u_{i} \mid q\left(u_{i-1}\right) ; \lambda_{L}\right)
$$

with $q\left(u_{i-1}\right)$ representing the state of arrival after the realisation of $u_{i-1}$.

For computing $\max _{k} P\left(\vec{c} \mid \overrightarrow{t_{k}}\right)$ we have assumed that there always exists a $\overrightarrow{t_{k}}$ comprising $I$ phone arcs corresponding to the phones $u_{k i}$ having the highest probability of emitting the classes $c_{i}$. If $P\left(\phi \mid U_{j}\right)$ represents the probability that a phone $U_{j}$ of the phone inventory is deleted whenever it is encountered in a transcription, then $1-P\left(\phi \mid U_{j}\right)$ is the probability that $U_{j}$ corresponds to a phonetic segment, and consequently:

$$
u_{k i}=\arg \max _{j}\left\{P\left(c_{i} \mid U_{j}\right)\left[1-P\left(\phi \mid U_{j}\right)\right]\right\}
$$

Furthermore, we expect that any acoustical realization of the transcription $\overrightarrow{t_{k}}$ will also exhibit a number of deleted phones. If we assume an average deletion probability $P(\phi)$ for these deleted phones, then we obtain that

$$
\max _{k} P\left(\vec{c} \mid \overrightarrow{t_{k}} ; \lambda_{L}\right) \approx \prod_{i=1}^{I} \max _{j}\left\{P\left(c_{i} \mid U_{j}\right)\left[1-P\left(\phi \mid U_{j}\right)\right]\right\} \prod_{l=1}^{L_{k}-I} P(\phi)
$$

with $L_{k}$ being the number of phone arcs in $\overrightarrow{t_{k}}$. If $P(\phi)$ is the mean phone deletion probability, the number of deleted phones can be written as $L_{k}-I=P(\phi) L_{k}$. Consequently:

$$
L_{k}-I=I \frac{P(\phi)}{1-P(\phi)}
$$

By introducing a factor $\alpha$ defined by:

$$
\log \alpha=\frac{P(\phi)}{1-P(\phi)} \log P(\phi)
$$

the expression for $\max _{k} P\left(\vec{c} \mid \overrightarrow{t_{k}} ; \lambda_{L}\right)$ can be rewritten as:

$$
\max _{k} P\left(\vec{c} \mid \overrightarrow{t_{k}} ; \lambda_{L}\right) \approx \prod_{i=1}^{I} \alpha \max _{j}\left\{P\left(c_{i} \mid U_{j}\right)\left[1-P\left(\phi \mid U_{j}\right)\right]\right\}
$$

Finally, the probabilities $P\left(c_{i} \mid u_{i} ; \lambda_{L}\right)$ can be derived from the outputs of the phonetic classification network computed for the different phones of the training set. However, if every acoustic realization of $U_{j}$ is assumed to have the same phonetic property $C_{j}=$ $c\left(U_{j}\right), P\left(c_{i} \mid u_{i} ; \lambda_{L}\right)$ can be approximated by a delta-function. As this approximation did not degrade the performance of our system, it was used in all reported experiments. 


\section{Conclusion}

Taking all the preceding remarks into consideration, we are looking for the couple $(\vec{s}, \vec{u})$ maximizing the expression:

$$
P=\prod_{i=1}^{I} P\left(s_{i} \mid s_{i-1}, \vec{v}_{s i} ; \lambda_{A}\right) \sum_{c_{i}} P\left(c_{i} \mid \vec{x}_{s i} ; \lambda_{L}, \lambda_{A}\right) \frac{P\left(c_{i} \mid u_{i} ; \lambda_{L}\right) P\left(u_{i} \mid q\left(u_{i-1}\right), \vec{t}\right)}{\alpha \max _{j} P\left(c_{i} \mid U_{j}\right)\left[1-P\left(\phi \mid U_{j}\right)\right]}
$$

with $\left(U_{1}, \cdots, U_{J}\right)$ representing the phone inventory and $q\left(u_{i-1}\right)$ the state of arrival in the state-transition model after having pronounced $u_{i-1}$

Obviously, if the probabilities $P\left(c_{i} \mid u_{i}\right)$ are approximated by delta-functions the sum on the right-hand side is reduced to a single term. We would also like to remark that the maxima in the denominator can be calculated in advance. Consequently, they do not represent a computational load to the algorithm.

\section{System Description}

Based on the mathematical expression (2), we have conceived the automatic segmentation and labelling system depicted in figure 2. The speech signal is analysed by means of an auditory model and subsequently segmented by an initial segmentation algorithm. Candidate phonetic segments are then constructed by merging up to 4 consecutive initial segments. Two neural networks are used to estimate the posterior phonetic segment $P\left(s_{i} \mid s_{i-1}, \vec{v}_{s i} ; \lambda_{A}\right)$ and the phonetic class probabilities $P\left(c_{i} \mid \vec{x}_{s i} ; \lambda_{L}, \lambda_{A}\right)$ of the hypothesized segments. Taking these probabilities into account, the speech is aligned with a state-transition model, derived from the transcription of the utterance.

Before describing the different building blocks in more detail, we would like to emphasize that different parts of the system include MLP's which are used as posterior probability estimators. This is justified by the fact that Richard and Lippmann ([Richard \& Lippmann 1991]) and others have proved that under ideal circumstances (enough training examples, enough free parameters, 1 output per class to be learnt), a MLP will indeed compute these probabilities after an appropriate training. 


\subsection{The Phonetic Expansion}

If the transcription of the sentence is a sequence of phonemes (as it is in the case of a citation, a phonotypical and an auditory transcription), this transcription has to be translated into a phone sequence. To accomplish this, each phoneme is represented by a state-transition model of one of the types represented in figure 3. Each transition Figure 3 is characterized by a transition probability and an emitted phone. The deletions of particular phones in the realization of a phoneme are accommodated by the so called deletion arcs (indicated by a $\phi$ ). The number of states is determined by the maximum length realization of the phoneme. For example, the voiced stop /d/, as in $\mathbf{d} a y$, may consist of a voiced occlusion $/ \mathrm{dcl} /$ and a burst / $d /$ (notation taken from TIMIT). Another example is the aspirated stop /th/ of eigth which may be modelled as an occlusion followed by a burst followed by an aspiration. Most phonemes however are represented by just one phone, e.g. the $/ a a /$ (as in mom).

Using the above phoneme models, the phonetic transcription is expanded to a large state-transition model (figure 1) representing a number of phone strings which are consistent with this transcription. Even if the phonetic transcription already is a phone string, it is still converted to a state-transition model by means of 2-state models (type (a) in figure 3). The deletion arcs can then accommodate for phones which were indicated by the human labeller, but which are not well reflected in the acoustical observations.

Note that the citation, the phonotypical and the auditory phonemic transcriptions may not incorporate acoustical events such as glottal stops. These glottal stops are therefore automatically inserted during the expansion on the basis of a simple pronunciation rule. We have assumed that the same glottal stop is to be inserted in front of any vowel which is preceded by a silence or another vowel. An alternative would have been to include a dedicated glottal stop as the initial phone of every vowel model.

Obviously, the phoneme expansion strategy applied in our system is much less sophisticated than the one advocated in [Angelini et al 1993, Brugnara et al 1993, Ljolje \& Riley 1991] which is based on trained pronunciation networks. However, our strategy has the advantage of requiring only a very limited amount of phonotactic knowledge, and little or no training (only the deletion probabilities have to be learnt), and last but not least, it does not require any manually labelled data to learn the most common 
pronunciations.

\subsection{The Auditory Model}

The signal is analysed by means of an auditory model [Martens \& Van Immerseel 1990, Van Immerseel \& Martens 1992]. The advantages of auditory based representations, especially under adverse conditions, have been demonstrated in [Anderson 1993, Martens \& Van Immerseel 1990, Meng \& Zue 1990, Meng \& Zue 1991, Seneff 1990, Van Immerseel 1993, Vereecken \& Martens 1995]. The auditory model used in this system incorporates an auditory filter bank, a bank of hair-cell models emphasizing the transitions at the phonetic boundaries, and a bank of envelope extractors measuring the envelopes of the hair-cell outputs in the different channels of the model. Every $10 \mathrm{~ms}$, the auditory model generates an acoustic observation vector $\vec{o}_{n}$ ( $n$ is the discrete time index). Each vector consists of an auditory spectrum (20 parameters), a voicing evidence, and a fundamental frequency (if there is enough voicing evidence). In addition to that, an energy function is computed by accumulating the hair-cell output envelopes across the different channels. As the auditory model is a running-time model, it was no problem to sample this function at a rate of $0.5 \mathrm{kHz}$. Its samples $E(n)$ thus represent energies at multiples of $2 \mathrm{~ms}$.

\subsection{Initial Segmentation}

As the probabilities in (2) imply segmental models, the Viterbi-search has to operate according to a stochastic segment model approach [Ostendorf \& Roukos 1989]. In order to speed-up this process, the search was constrained by introducing a set of potential phone boundary locations $\vec{b}=\left(b_{1}, \cdots, b_{K}\right)$ (with $K<N$ ) emerging from an initial segmentation which starts by identifying major changes (landmarks) in the acoustic signal.

The identification of acoustic landmarks is based on a very fast temporal analysis of the energy function $E(n)$ emerging from the auditory model. At each discrete time $n$, representing multiples of $2 \mathrm{~ms}$, the left $\left(D_{l}(n)\right)$ and right $\left(D_{r}(n)\right)$ derivatives are defined as the slopes of the best linear regressions of $E(n)$ in the time windows $(n-W, n)$ and $(n, n+W)$ respectively. The width $W$ of the regression windows is a free parameter of 
the algorithm. Given the derivatives, a curvature function $C(n)$ is computed as follows:

$$
C(n)= \begin{cases}D_{r}(n)-D_{l}(n) & \text { if } D_{r}(n)>D_{l}(n) \text { and } D_{r}(n) * D_{l}(n) \geq 0 \\ \max \left(D_{r}(n),-D_{l}(n)\right) & \text { if } D_{r}(n)>D_{l}(n) \text { and } D_{r}(n) * D_{l}(n)<0 \\ 0 & \text { if } D_{r}(n)<D_{l}(n) .\end{cases}
$$

This curvature function detects sudden increases in the derivative and at the same time reduces the effect of fluctuations in $E(n)$ which are due to the fundamental frequency, and which often occur in voiced sounds of male speakers. An example of a speech signal and the corresponding $E(n)$ and $C(n)$ (of a vowel) is depicted in figure 4. This picture also includes the curvature function $C^{\prime}(n)$ which would have been obtained by selecting $D_{r}(n)-D_{l}(n)$ whenever $D_{r}(n)>D_{l}(n)$.

The landmark generation algorithm now searches for significant maxima in $C(n)$. The sum of the $C(n)$ encountered in the interval bounded by the minima surrounding such a maximum, constitute the preliminary evidence of the landmark. If it is found closer than $\Delta_{t}$ to the previously retained landmark, the new landmark is not retained but its evidence is added to that of the last retained landmark. If the distance to the previous landmark however is larger than $\Delta_{t}$, the new landmark is retained provided its evidence is larger than some threshold $\delta_{1}$. This strategy will force the retained landmarks to the start of a transition region (see e.g. the third dashed line on figure $4 \mathrm{c}$ ).

The threshold $\delta_{1}$ controls the amount of landmarks being generated. The parameters of this landmark analysis were set experimentally. The purpose was to minimize the number of non-phonetic boundaries (insertions) in $\vec{b}$ while fixing the number of phonetic boundaries not present in $\vec{b}$ (deletions)to some small value. By evaluating the performances on our Flemish database (see further), we obtained $\delta_{1}=0.125$ and $\Delta_{t}=8 \mathrm{~ms}$. The length of the regression window was fixed to $12 \mathrm{~ms}$. The same parameter values were maintained when applying the algorithm to other databases. The dotted lines on figure 4 show the landmarks being generated using these settings. Apparently, $C(n)$ yields less insertions than $C^{\prime}(n)$.

In a landmark elimination stage, each acoustic landmark is characterized by a set of features describing properties of the speech in the immediate vicinity of that landmark. A 2-layer MLP with 10 hidden units and 1 output is trained to estimate the probability that the landmark is a true phonetic boundary given these features. If this probability is 
below a particular threshold (experimentally set to 0.175), the landmark is withdrawn. To preserve as much as possible the speaker, vocabulary and language independent nature of the acoustic landmark analysis, the number of features is kept limited to 12: the evidences of the examined, the previous and the next landmark as they emerge from the landmark analysis, the values of $E(n)$ at these landmark positions, the distances between these landmarks, the sum of the increases and the sum of the decreases in the energy per channel between the frames around the landmark.

\subsection{Phonetic Segmentation}

This module is intended to compute the probability $P\left(s_{i} \mid s_{i-1}, \vec{v}_{s i}, \lambda_{A}\right)$ that boundary $s_{i}$ is a phonetic boundary given that $s_{i-1}$ is the preceding phonetic boundary and given the acoustic evidence. This probability is estimated by a MLP with one output, 12 hidden units and 13 inputs. These inputs are obtained as follows:

- The mean auditory spectrum (20 channels) of segment $\left(s_{i-1}, s_{i}\right)$ is represented by 5 numbers, namely the accumulated contributions to this spectrum observed in 5 disjunct sets of 4 consecutive channels.

- A difference spectrum (also represented by 5 numbers) is computed as follows. If no initial boundaries occur inside $\left(s_{i-1}, s_{i}\right)$, the difference spectrum is assumed zero, otherwise it is the difference between the mean spectra of subsegments $\left(s_{i-1}, b^{*}\right)$ and $\left(b^{*}, s_{i}\right)$ with $b^{*}$ being the initial boundary with the highest evidence encountered in $\left(s_{i-1}, s_{i}\right)$.

- The remaining 3 parameters are the segment duration and the initial boundary evidences of $b^{*}$ and $s_{i}$ as they emerge from the initial segmentation. If no $b^{*}$ was found, the corresponding evidence is assumed zero.

\subsection{Phonetic Classification}

The automatic segmentation and labelling uses intermediate phonetic classes to be derived from the acoustic signal. To establish an appropriate class set, the following considerations must be taken into account: 
1. In order to obtain one likely phone sequence $\vec{u}$ given the acoustic signal and the transcription model $\vec{t}$, there should be one likely $\vec{c}$ emerging from the acoustic models. If two phones of the same class are merged, it is likely that the phonetic classification network will indicate this segment as a member of that class as well. For finding the correct solution the system then has to rely on the phonetic segmentation probabilities. It is therefore safer to pursue that subsequent phones belong to different classes.

2. If the number of classes becomes larger, the classes will become more confusable and the acoustic models will produce several competing $\vec{c}$ s and corresponding $\vec{u}$ 's. Therefore, it helps if the phonetic classes are less confusable.

3. If we want to obtain a system which is easy to adapt from one language to another, the phonetic classes should be related to the articulatory description of the speech sounds.

To determine the 'best' set of phonetic classes, we have examined the confusability and the amount of discriminative boundaries (i.e. boundaries between phones belonging to different phonetic classes) implied by different class sets. We have done this for several sets of classes describing the manner and place of articulation of the Dutch phones. We started from 12 classes: high front vowels, medium front vowels, central vowels, high back vowels, low back vowels, nasals, liquids and glides, occlusions of voiced stops, voiced fricatives, unvoiced fricatives, bursts of stops, silences and occlusions of unvoiced stops. Then we gradually reduced the number of classes to 3 . In addition, the complete set of 54 phones was used as a class set as well.

The percentage of discriminative boundaries increases from $80 \%$ to $100 \%$ if the number of classes is increased from 3 to 54 (the total number of phones) (figure 5). The Figure 5 corresponding classification accuracy that can be obtained using a MLP decreases from $96 \%$ to $67 \%$.

If we assume that both sources of errors are equally important, the global performance of the class sets as a function of the number of classes (figure 5) reaches an optimum for 5 classes. This is in agreement with the findings of Dalsgaard et al. [Dalsgaard et al 1991a, Dalsgaard et al 1992] that 5 distinctive features are sufficient to control an automatic 
segmentation and labelling system. In all our experiments, the following 5 phonetic classes were used: vowel, consonant-sonorant, fricative, burst and silence.

The probabilities $P\left(c_{i} \mid \vec{x}_{s i} ; \lambda_{L}, \lambda_{A}\right)$ are again computed by a two-layer MLP. This neural network has 39 inputs, 30 hidden units and 5 outputs. The inputs are related to the mean spectrum and voicing evidence, and the duration of the segment, and the spectra observed in the $20 \mathrm{~ms}$ left and right context of the segment.

\subsection{Viterbi Alignment}

In the Viterbi-alignment module, the speech segments are aligned with the statetransition model that was derived from the transcription of the utterance. The search maximizes the expression

$$
P=\prod_{i=1}^{I} P\left(s_{i} \mid s_{i-1}, \vec{v}_{s i}, A\right) \sum_{c_{i}} P\left(c_{i} \mid \vec{x}_{s i}, A\right) \frac{P\left(c_{i} \mid u_{i}\right) P\left(u_{i} \mid q\left(u_{i-1}\right), \vec{t}\right)}{\alpha \max _{U_{j}} P\left(c_{i} \mid U_{j}\right)\left[1-P\left(\phi \mid U_{j}\right)\right]}
$$

It performs a search in a 2-dimensional trellis with the initial segment boundaries $(\vec{b})$ on the $\mathrm{x}$-axis and the states of the state-transition model $(\vec{t})$ on the $\mathrm{y}$-axis. Constraining the Viterbi-search to the initial segment boundaries reduces the number of time instances to consider to about $N / 4$ (with $N$ the total number of frames). Furthermore, the number of transitions arriving in a node of the trellis is reduced to 5 ( 1 deletion arc with $s_{i}=s_{i-1}$ and 4 phone arcs with $\left.s_{i}-s_{i-1}=1, \cdots, 4\right)$. To increase the speed of the search even further, only the trellis nodes in a belt around the diagonal are investigated.

\section{$5 \quad$ Training and Evaluation}

The multi-layer perceptrons incorporated in the initial segmentation, the phonetic segmentation and the phonetic classification modules, were initially trained on 10 minutes of manually labelled phonetically rich speech provided by 20 training speakers (Flemish continuous speech). The Flemish continuous speech was recorded in an anechoic chamber, bandlimited to $4 \mathrm{kHz}$ and sampled at $10 \mathrm{kHz}$. The sentences were selected from a set of 130 phonetically balanced sentences. The transition probabilities in the phoneme models were obtained by performing a first automatic annotation step using auditory phonetic transcriptions. In this step, initial values of 0.05 for all the deletion probabili- 
ties in the phoneme models were employed. The final values of the deletion probabilities were then retrieved from the best state sequences through the state-transition models. A further adaptation of the transition probabilities did not improve the segmentation and labelling performance anymore.

Once the system was trained, it was tested on the training corpus and on an independent test set of 10 new speakers each uttering 13 phonetically rich sentences. The results of these tests are described in terms of segmentation and labelling errors. To evaluate these errors, the automatic segmentation and labelling is aligned with the manual one using the Dynamic Time Warping (DTW) procedure described in [Martens \& Depuydt 1991]. Due to the applied cost function, "gross" errors (defined in the SAM-project, e.g. [Kvale 1993], as automatic segments having no overlap with their corresponding manual segments) are very unlikely to emerge from this alignment. Therefore, we distinguish three kinds of segmentation errors ("segm"): deletions ("del", a manual segment boundary was omitted), insertions ("ins", an automatic boundary was inserted between two manual boundaries) and boundary deviations ("far", the placement of the automatic and the corresponding manual boundary differ by more than $20 \mathrm{~ms}$ ). The same boundary deviation error criterion is also used by others [Dalsgaard 1992, Kabré et al 1991, Sørensen \& Dalsgaard 1989, Svendsen \& Kvale 1990, van Erp et al 1989]. The labelling errors ("sub") indicate the number of times that an automatically labelled phone differs from the manual one to which it was assigned by the DTW-process. The total error ("tot") is the sum of the segmentation and the labelling errors. All errors are specified in percent, relative to the number of phones occurring in the manual labellings.

The results obtained for the training and the test corpus of the Flemish database are listed in table 1. Notice that experiments [Cosi et al 1991, van Erp et al 1989] have Table 1 shown that different manual labellers also disagree over more than $20 \mathrm{~ms}$ in about $10 \%$ of the cases. 


\section{Adapting to a New Task}

The labelling of new databases is straightforward. First of all, if a new language is involved, one needs the inventory of phonemes existing in that language and a set of phoneme models representing the maximum length phone sequence realizations of these phonemes. Subsequently, one also has to provide the phonetic class specifications of the phones encountered in the phoneme models. As the phonetic classes are based on the manner of articulation, they should be easy to retrieve from the literature.

In a first step, the new data are automatically segmented and labelled using the Flemish baseline system. Note that in this stage, initial values of 0.05 are used for the deletion probabilities in the phoneme models. The obtained alignments then provide the observations required for updating the phonetic classification network and thus improving the acoustic models. The other MLP's were left unaltered, as it was found that retraining them did not improve the ultimate performance of the system. In a second phase, the transition probabilities of the statistical phoneme models are automatically derived from the best state sequence through the phonetic transcription model, obtained using the updated phonetic classification network. The system has then completely adapted to the new task.

\section{Performance Evaluation}

To assess the portability of our automatic labelling system to other languages, several databases were segmented and labelled. In a first step, five isolated word databases (a Dutch, a French, an American English, a Spanish and a Korean one) were processed. To assess the performance of our system on continuous speech data and to enable a comparison of our results with those reported by others, additional experiments were performed on the TIMIT database and on the English, Danish and Italian passages of the EUROM0 database. In all experiments, a manual segmentation and labelling of the utterances was available for evaluation purposes. 


\subsection{Isolated Word Corpora}

Each database consisted of a few thousand words uttered by a single speaker (but a different speaker for each language) and sampled at $10 \mathrm{kHz}$. These databases were used for the development of a segment concatenation based speech synthesis system. Each word list was thus designed as to contain all the diphones and triphones required for the synthesis of speech in the target language. The speech signals were aligned with the citation phonemic transcriptions of the words. The same data were used for adapting and for testing the automatic segmentation and labelling system. As it was the purpose to label each database as accurately as possible, this is a valid approach. The results of a comparison between the automatic and the manual segmentation and labelling are listed in table 2. The listed results clearly demonstrate the feasibility of our annotation approach.

The error rates are quite stable across the different languages. When comparing to manual labelling experiments [Cosi et al 1991, van Erp et al 1989], the number of boundary deviations is certainly acceptable. The number of insertions and substitutions can be neglected. The Romanic languages seem to yield more boundary deviations than the other languages. This is in agreement with the findings of [van Erp et al 1989].

\subsection{Results on TIMIT}

Our annotation strategy was tested on the core test portion of the TIMIT database (only the 5 sx-sentences per speaker). Although we used the complete phone set of the TIMIT database in our phone inventory, we evaluated our results with respect to the restricted phone set of 48 labels (phone set proposed by Lee and Hon [Lee \& Hon 1989]) in order to allow a fair comparison of the substitution errors with those reported by others.

In a first experiment, the system is adapted to the new task using a portion of the training set of TIMIT. The adaptation is performed on the basis of the citation phonemic transcriptions. Experiments have pointed out that about 100 utterances (i.e. about 3 to 4 minutes of speech) are sufficient to adapt the system to the new task. Both the baseline system and the adapted system were tested on the same test set, with the citation phonemic transcriptions as the lexical input. The results of these tests are listed in table 3.

Table 3 
In the second experiment, the adapted system (from above) was used to align the speech with the manual label sequence. During this alignment, phone deletions were permitted, but when a deletion was observed, an extra phone was inserted automatically. If a phone was deleted, it was normally supposed to coincide with the initial $10 \mathrm{~ms}$ of the succeeding (and detected) phone, but in the case of an intervocalic consonant it was supposed to coincide with the final $10 \mathrm{~ms}$ of the preceding vowel. Using this insertion strategy, there were no deletions, insertions nor substitutions anymore. The number of correctly placed boundaries is equal to $76 \%$. Observe that the baseline system yields $75 \%$ correctly placed boundaries. The fact that the two system performances are so close to one another indicates that the segment generation and the segment classification play dominant roles in the annotation task: the initial segmentation and the phonetic/nonphonetic classification do not differ for the two systems.

In the third experiment the system was retrained (using about 200 training utterances) using the manual segmentation and labelling provided with the utterances. Again, the (manually) retrained system was evaluated on the core test set using citation phonemic transcriptions. The results (table 4) indicate that a gain of about $5 \%$ in the total Table 4 error rate can be obtained by using manually annotated utterances during the training.

Brugnara et al. [Brugnara et al 1993] performed an experiment that is equivalent to our first test. They obtained $42.7 \%$ segmentation errors (2.7\% deletions, $11.3 \%$ insertions and $28.7 \%$ boundary deviations) and $13.9 \%$ substitutions with a system based on HMM's obtained by an embedded training using the citation phonemic transcriptions as lexical inputs. Aligning with the manual labels (our second experiment) resulted in $73.4 \%$ correctly placed boundaries. Only if the HMM training was performed using the manual label strings (our third experiment), the number of boundary deviations could be reduced to $16.4 \%$ (yielding a segmentation error rate of $30.4 \%$ ).

Angelini et al. [Angelini et al 1993] also performed several experiments on the TIMIT database. However, these experiments cannot be compared directly to ours, as the manual label strings were used to train the HMM's and to synthesise a pronunciation network for each word (we only use the standard word pronunciation for each word). They obtain the following results: $22.5 \%$ segmentation errors (5.6\% deletions, $3.1 \%$ insertions, $13.8 \%$ boundary deviations) and $13.5 \%$ substitutions. 
Ljolje and Riley [Ljolje \& Riley 1991] propose another labelling system. It comprises continuous density variable duration HMM's, a trigram phonotactic model for constraining the phone sequence, gamma distributions for the phone durations, and different topologies for modelling phones in different contexts. Furthermore, the HMM's were trained using the existing TIMIT manual labels (although disregarding the label positions). The manual labels of 3360 transcribed utterances were also used to train a phone realization tree for generating the most likely phone sequences, given the orthographic representations of the utterances. With that system, the authors obtain a phone sequence estimation error rate of about $12 \%$ ( $10 \%$ deletions and substitutions and $2 \%$ insertions), and a boundary deviation rate of about 20\% (differences of more than $17 \mathrm{~ms}$ between the automatic and the manual boundaries were considered as boundary deviations). As the exact number of deletions is not indicated in [Ljolje \& Riley 1991] or [Riley 1991], we assume an even balance between the deletion and the substitution errors. In that case, the total segmentation error rate is $27 \%$.

As the last two systems require a large amount of manually annotated utterances to train detailed acoustic models and complex pronunciation networks, they are not easily ported to another language. One may even question their portability to another task within the same language.

\subsection{Results on EUROMO}

We also performed evaluation experiments on the English, Danish and Italian passages of the EUROM0 database. For each language about 8 minutes of speech (four speakers: 2 male and 2 female) were available. We started (mode 1) by evaluating the baseline system (trained on the Flemish data and not adapted to the new languages). Then (mode 2), the data of three speakers was used to adapt the system, while the data of the remaining speaker was used for evaluation. Finally (mode 3), the speech of one speaker was used both to adapt and to evaluate the system. For each experiment, four tests were performed, i.e. each speaker was once used as the evaluation speaker. The input transcriptions for all the tests were phoneme sequences derived from the manual label sequence (as described in section 2.1). The results listed in table 5 represent the Table 5 mean error percentages of the four tests. As the glottal stops (automatically inserted 
during the phoneme expansion) were not marked in the reference (manual) segmentation and labelling, they were absorbed in the subsequent vowel before the evaluation was performed.

The results of the second and third experiment are quite comparable. Apparently, the speaker-dependent acoustic models (experiment 3) do not perform any better than the speaker-independent models (experiment 2). This is due to the insufficient amount of training data available for the adaptation of the speaker-dependent models. The results of experiment 1 demonstrate that the adaptation improves the annotation performance, and that this improvement is mainly characterized by a significant reduction of the number of deletion errors.

In a similar experiment, but with the manual label string as the lexical input Dalsgaard et al. [Dalsgaard et al 1991b] achieve 60.5\% correctly placed boundaries (within $20 \mathrm{~ms}$ ) on the English and $47 \%$ on the Italian set with a system that was trained on the Danish set. If we align the speech with the manual label string using our Flemish baseline system we obtain 76.6\% (English), 74.6\% (Danish) and 78.5\% (Italian) segment position accuracy. After adaptation (using the phonemic transcriptions derived from the manual label strings as lexical input), we obtain 77.5\% (English), 76.8\% (Danish) and $78.5 \%$ (Italian) correctly placed boundaries.

Kvale [Kvale 1993] also reports annotation experiment on EUROM0-data. Just as in

our second experiment, the annotation system was trained on 3 speakers (per language) and tested on the remaining speaker. During training and testing, the manual labels were used as lexical inputs. The main difference between this system and ours is that the system of Kvale performs a segmentation in phonemes. The reported phoneme boundary position accuracies (threshold of $20 \mathrm{~ms}$ ) were $86.1 \%$ for British English, 82.3\% for Danish and $84.5 \%$ for Italian.

\subsection{Detailed Analysis of the Segmentation Errors}

To illustrate some discrepancies between the manual and the automatic annotations, we have included some pictures showing the speech signal, its auditory spectrogram and the total energy function emerging from the auditory model. The vertical lines through the figures indicate manually placed boundaries. The bottom lines indicate automatically 
generated boundary positions. The time (horizontal axis) is always indicated in ms.

For the isolated word databases, the number of deletions is rather small, but there are some systematic errors. For the American English and the French databases for instance, more than $30 \%$ of the deletions appear to be glottal stops. For Dutch on the other hand, most of the deleted labels were low energy word initial $b$ and $d$ phones (voiced occlusions of the corresponding stops), as in the example of figure 6 (top). More than half of the deletions occurring in the Spanish database can be attributed to the voiceless stop $t$ (e.g figure 6 , bottom).

A number of boundary deviations for the French database is caused by the fact that the automatic system seems to use another criterion than the human labeler to position the start of an utterance-initial fricative (figure 7, top). Another reason for the discrepancy between the languages was due to questionable glide-vowel manual boundary positions (figure 7, bottom) encountered in the Romanic databases.

The segmentation and labelling of continuous speech is a much more difficult task and yields much more deletion errors. For TIMIT, almost $30 \%$ of the deletions originate from the liquids and the glides, while the occlusions of the stops and the pauses account for another $25 \%$ of the deletion errors. For the EUROM0 databases, more than half of the deletions (and all of the insertions) can be credited to the occlusions and the bursts of stops. For the English part, some other frequently deleted labels are the liquids $(r$ and $l$ ). For Danish, it is often the $D$, and for Italian the $v$ that is deleted. However, it should be noted that there is often no evidence of the phones in the signal either, e.g. the $h h$ (TIMIT) and the $D$ (English EUROM0)in fig 8.

Figure 8

Obviously, some deletions are due to limitations of the automatic system. For instance, the segmentation algorithm cannot detect segments that are shorter than 8 ms. Short bursts are therefore often deleted. Furthermore, if no clear transition exists between phones, one of the phones may be deleted (fig 9, top). Most of these deletions Figure 9 could possibly be eliminated by means of a post processing stage.

Some segmentation errors emerge from an incorrect transcription of the utterance (e.g. the citation phonemic transcription). An example extracted from the TIMIT database is shown in figure 9 (bottom). As the citation phonemic transcription was ao $r$ $w$ er, the automatic segmentation and labelling system is asked to search for 4 segments. 
Apparently, the human labeller did not find enough evidence for a separate $r$ and used the diphone axr to model the vowel and the subsequent $r$. The automatic system did find enough boundaries to put two phones between the first and the second vowel, and labelled them as $r$ and $w$ respectively.

The number of segment boundary deviations is most probably an overestimation of the real error rate (figure 10). Because the baseline system was trained on manually labelled utterances, the segmentation module is biased towards the strategy employed during the segmentation of these utterances. This strategy however can be different from that employed in other databases. As an example, the vowel-sonorant and vowel-fricative boundaries in EUROM0 are usually placed earlier than in the Flemish database. The reverse is true for the sonorant-vowel transitions. This kind of knowledge could easily be used to reduce the error rates for these databases. However, as our intention is not to imitate the manual labeller at all costs, but to provide a consistent and accurate annotation performing well across languages, we did not want to introduce such kinds of post processing schemes.

\section{Computer-Assisted Annotation}

Our annotation system has proven to be a very valuable tool for the development of multi-lingual text-to-speech systems. For each language, one has to record an isolated word database from which to retrieve the speech units of that language, and a read continuous speech database from which to retrieve appropriate duration models for these speech units. If phonotypical transcriptions for the recorded utterances are provided, our system produces (after adaptation) accurate automatic annotations. Nevertheless, this annotation may require further improvements. Therefore, the automatic annotation system was incorporated in a manual labelling tool. After performing an automatic annotation, the human labeller can either displace erroneous boundaries or perform a constrained automatic annotation pass during which the alignment is forced to position certain labels at prescribed positions. It is our experience that the manual annotation of a speech database can now be performed about 20 times faster than before. 


\section{Conclusion}

We have shown that it is possible to build an automatic segmentation and labelling system that can be trained on one task (e.g Flemish continuous speech), and automatically adapted to a new task (e.g. English continuous speech) without requiring any manually labelled utterances which are representative for this new task. It has been demonstrated that the adapted system performs equally well or even better than systems reported in the literature, and which were specifically trained for that task.

Obviously, if manually segmented and labelled data is available for the new task, this data can also be used to retrain the system. By doing this the segmentation and labelling performance can be improved even further. If the transcriptions do not perfectly match the corresponding speech signals, like in the case of citation or phonotypical phonemic transcriptions, the segmentation and labelling is of course less accurate than in the case auditory or manual-labelling transcriptions are used.

The adaptation to a new language involves the specification of the phoneme inven-

tory, a description of the maximum length phone realisations of these phonemes, the registration of a few minutes of speech with the corresponding transcription, and an hour of CPU-time on a 20 MFlops workstation to adapt the acoustic models.

\section{Acknowledgment}

We would like to thank the reviewers for their valuable remarks and suggestions concerning the presentation of our work.

\section{References}

[Anderson 1993] T. R. Anderson (1993), "A Comparison of Auditory Models for Speaker Independent Phoneme Recognition", Proceedings of ICASSP-93, Vol 2, pp. 231-234.

[Angelini et al 1993] B. Angelini, F. Brugnara, D. Falavigna, D. Giuliani, R. Gretter and M. Omologo (1993), "Automatic Segmentation and Labeling of English and Italian Speech Databases", Proceedings of Eurospeech-93, pp. 653-656. 
[Arai et al 1990] K. Arai, Y. Yamashita, T. Kitahashi and R. Mizoguchi (1990), "A Speech Labeling System Based on Knowledge Processing", Proceedings of ICSLP-90, pp. 10051008 .

[Barry \& Fourcin 1992] W. J. Barry and A. J. Fourcin (1992), "Levels of Labelling", Computer Speech and Language, Vol. 6, pp. 1-14.

[Brugnara et al 1993] F. Brugnara, D. Falavigna and M. Omologo (1993), "Automatic segmentation and Labeling of speech based on Hidden Markov Models", Speech Communication, Vol. 12 no. 4, pp. 357-370.

[Cosi et al 1991] P. Cosi, D. Falavigna and M. Omologo (1991), "A Preliminary Statistical Evaluation of Manual and Automatic Segmentation Discrepancies", Proceedings of Eurospeech-91, pp. 693-696.

[Dalsgaard 1992] P. Dalsgaard (1992), "Phoneme Label Alignment using Acoustic-Phonetic Features and Gaussian Probability Density Functions", Computer Speech and Language, Vol. 6, pp. 303-329.

[Dalsgaard et al 1991a] P. Dalsgaard, O. Andersen and W. Barry (1991), "Multi-Lingual Label Alignment using Acoustic-Phonetic Features Derived by Neural-Network Technique", Proceedings of ICASSP-91, pp. 197-200.

[Dalsgaard et al 1991b] P. Dalsgaard, O. Andersen and W. Barry (1991), "Multi-Lingual Acoustic-Phonetic Features for a Number of European Languages", Proceedings of Eurospeech-91, pp. 685-688.

[Dalsgaard et al 1992] P. Dalsgaard, O. Andersen, W. Barry and R. Jørgensen (1992), "On the Use of Acoustic-Phonetic Features in Interactive Labelling of Multi-Lingual Speech Corpora", Proceedings of ICASSP-92, pp. 549-552.

[Grice et al 1989] M. Grice, W. J. Barry and A. Fourcin (1989), "Specification of EUROM0 Assessment", Appendix B: Part 2, in Support available from SAM-project for other ESPRIT speech and language work, SAM-document G001/B/2.

[Kabré et al 1991] H. Kabré, G. Pérennou and N. Vigouroux (1991), "A Non-Linear Filtering Method Applied to Automatic Segmentation of Multilingual Speech Corpora", Proceedings of Eurospeech-91, pp. 689-692. 
[Kvale 1993] K. Kvale (1993), Segmentation and Labelling of Speech, PhD Thesis, Institutt for Teleteknikk, Trondheim, Norway.

[Lamel et al 1986] L. Lamel, R. Kassel and S. Seneff (1986), "Speech Database Development: Design and Analysis of the Acoustic-Phonetic Corpus", Proc. DARPA Speech Recognition Workshop, pp. 100-109. (also distributed with the TIMIT CD-ROM)

[Lee \& Hon 1989] K. F. Lee and H-W. Hon (1989), "Speaker-Independent Phone Recognition Using Hidden Markov Models", IEEE Trans. on Acoustic, Speech, and Signal Processing, ASSP 37 no. 11, pp. 1641-1648.

[Leung \& Zue 1984] H. C. Leung and V. W. Zue (1984), "A Procedure for Automatic Alignment of Phonetic Transcriptions with Continuous Speech", Proceedings of ICASSP-84, pp. 2.7.1-2.7.4.

[Ljolje \& Riley 1991] A. Ljolje and M. D. Riley (1991), "Automatic Segmentation and Labeling of Speech", Proceedings of ICASSP-91, pp. 473-476.

[Martens \& Depuydt 1991] J.-P. Martens and L. Depuydt (1991). "Broad Phonetic Classification and Segmentation of Continuous Speech by means of Neural Network and Dynamic Programming", Speech Communications, Vol. 10, pp. 81-90.

[Martens \& Van Immerseel 1990] J.-P. Martens and L. Van Immerseel (1990). "An Auditory Model based on the Analysis of Envelope Patterns", Proceedings of ICASSP-90, pp. 401-404.

[Meng \& Zue 1990] H. M. Meng and V. W. Zue (1990), "A Comparative Study of Acoustic Representations of Speech for Vowel Classification using Multi-Layer Perceptrons", Proceedings of ICSLP-90, pp. 1053-1056.

[Meng \& Zue 1991] H. M. Meng and V. W. Zue (1991), "Signal Representation Comparison for Phonetic Classification", Proceedings of ICASSP-91, pp. 285-288.

[Ostendorf \& Roukos 1989] M. Ostendorf and S. Roukos (1989), "A Stochastic Segment Model for Phoneme-Based Continuous Speech Recognition", IEEE Transactions ASSP, Vol. 37, pp. 1857-1869.

[Richard \& Lippmann 1991] M. Richard and R. Lippmann (1991), "Neural network classifiers estimate Bayesian a posteriori probabilities", Neural Computation, Vol. 3, pp. 461-483. 
[Riley 1991] M. D. Riley (1991), "A Statistical Model for Generating Pronunciation Networks", Proceedings of ICASSP-91, pp. 737-740.

[Seneff 1990] S. Seneff (1990), "A Joint Synchrony/Mean-Rate Model of Auditory Speech Processing", In Readings in Speech Recognition, A. Waibel and K. F. Lee (Morgan Kaufmann), pp. 101-111.

[Sørensen \& Dalsgaard 1989] H. B. D. Sørensen and P. Dalsgaard (1989), "Multi-Level Segmentation of Natural Continuous Speech using Different Auditory Front-Ends", Proceedings of Eurospeech-89, Vol. 2, pp. 79-82.

[Svendsen \& Kvale 1990] T. Svendsen and K. Kvale (1990), "Automatic Alignment of Phonemic Labels with Continuous Speech", Proceedings of ICSLP-90, pp. 997-1000.

[Torkkola 1988] K. Torkkola (1988), "Automatic Alignment of Speech with Phonetic Transcriptions in Real Time", Proceedings of ICASSP-88, pp. 611-614.

[van Erp \& Boves 1988] A. van Erp and L. Boves (1988), "Manual Segmentation and Labelling of Speech", Proceedings of Speech-88, pp. 1131-1138.

[van Erp et al 1989] A. van Erp, C. Houben, B. Barry, M. Grice, L. J. Boë, G. Braun, P. Cosi, N. Dyhr, G. Pérennou, N. Vigouroux and D. Auteserre (1989), "A Unified Approach to the Labelling of Speech: First Multilingual Results", Proceedings of Eurospeech-89, Vol. 2, pp. 88-91.

[van Hemert 1985] J. P. van Hemert (1985), "Automatic Diphone Preparation", IPO Annual Progress Report, pp. 23-32.

[Van Immerseel 1993] L. M. Van Immerseel (1993), "Een functioneel gehoormodel voor de analyse van spraak bij spraakherkenning". PhD Thesis, University Gent, Belgium. (in Dutch)

[Van Immerseel \& Martens 1992] L. M. Van Immerseel and J.-P. Martens (1992), "Pitch and Voiced/Unvoiced Determination with an Auditory Model", J. Acoust. Soc. Amer., Vol. 91 no. 6 , pp. $3511-3526$.

[Vereecken \& Martens 1995] H. Vereecken and J.-P. Martens (1995), "Recognition of Noisy Speech using an Auditory Model", Proceedings of EUROSPEECH-95, pp. 1995-1998. 
[Wang et al 1990] H. D. Wang, G. Bailly and D. Tuffelli (1990), "Automatic Segmentation and Alignment of Continuous Speech Based on Temporal Decomposition Model", Proceedings of ICSLP-90, pp. 457-460. 


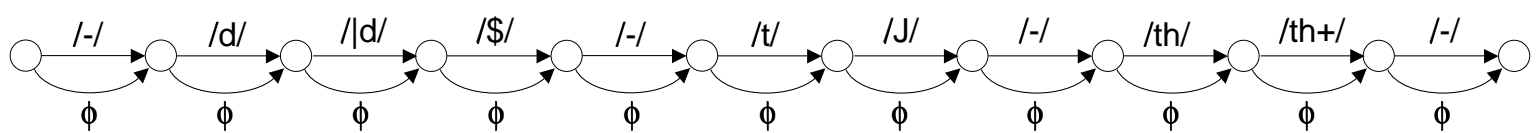

Figure 1: 


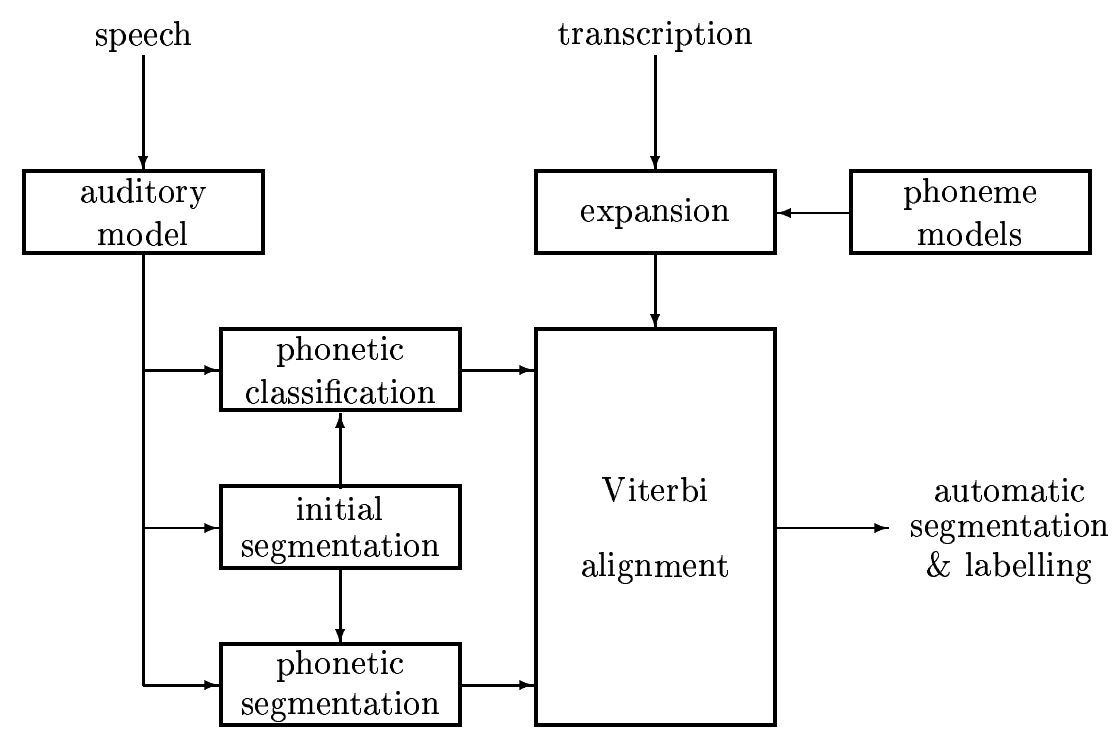

Figure 2: 


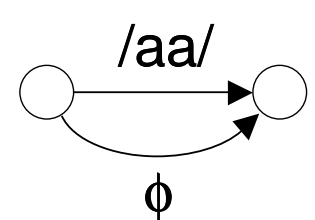

(a)

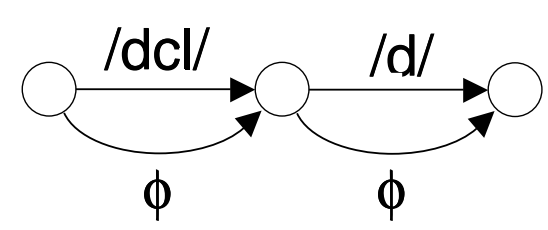

(b)

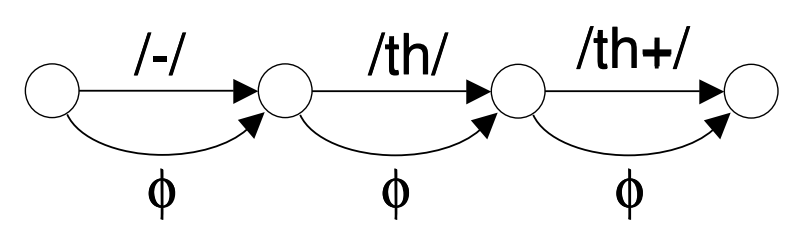

(c)

Figure 3: 

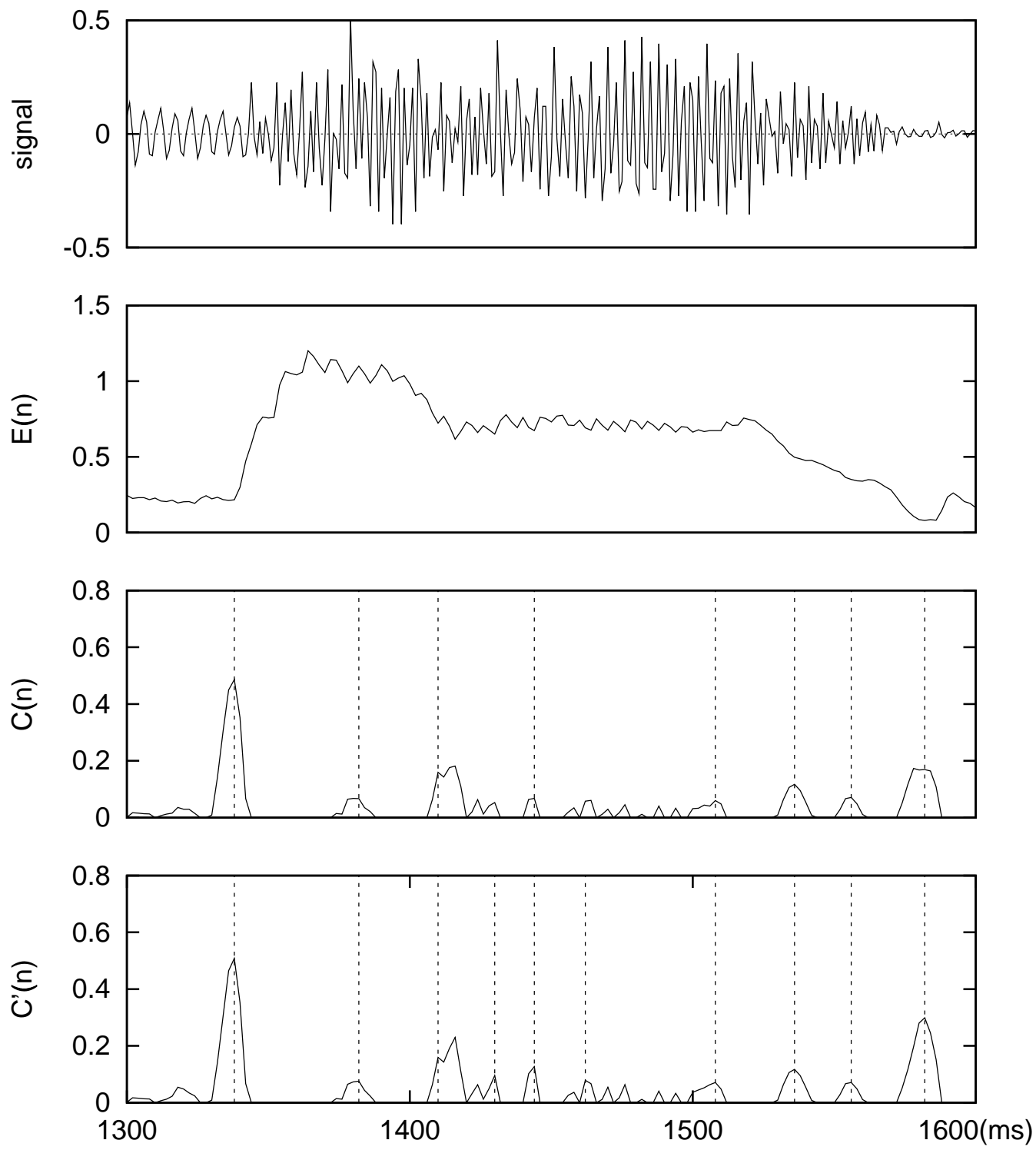

Figure 4: 


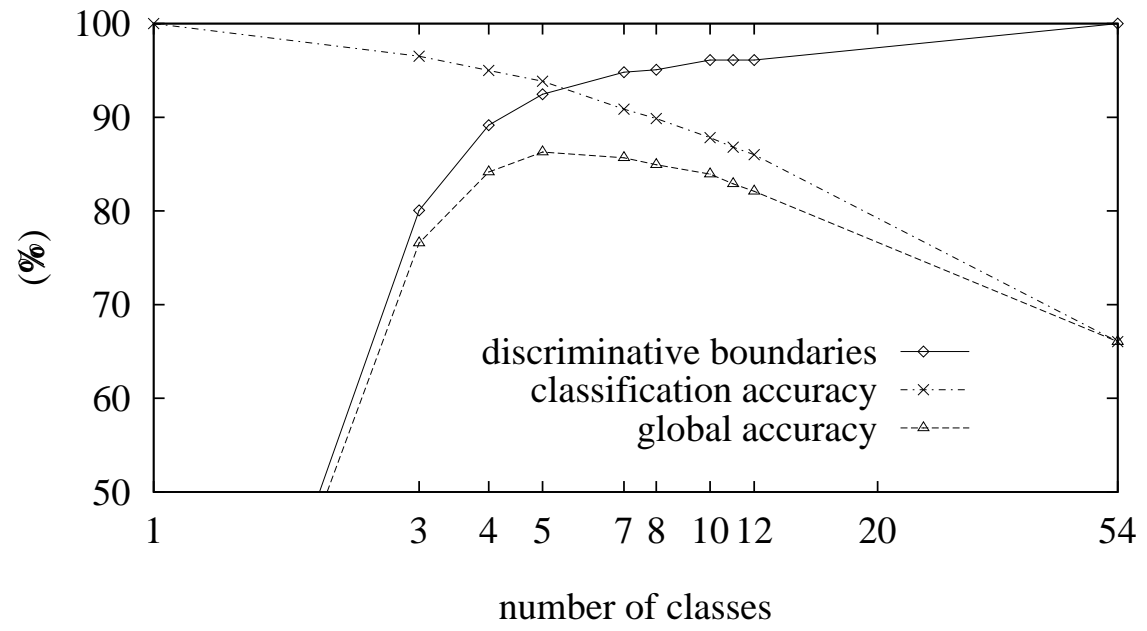

Figure 5: 


\begin{tabular}{|l|r|r||r|r|r|r|}
\hline & tot err & segm err & del & ins & far & sub \\
\hline train & 17.87 & 16.25 & 2.71 & 4.58 & 8.96 & 1.62 \\
\hline test & 19.38 & 17.33 & 2.37 & 4.55 & 10.42 & 2.04 \\
\hline
\end{tabular}

Table 1: 


\begin{tabular}{|l|r|r||r|r|r|r|}
\hline & tot err & segm err & del & ins & far & sub \\
\hline Dutch & 11.07 & 11.03 & 1.81 & 0.14 & 9.08 & 0.04 \\
\hline French & 13.67 & 13.66 & 0.43 & 0.14 & 13.09 & 0.01 \\
\hline Am. Eng. & 10.90 & 10.90 & 1.21 & 0.10 & 9.59 & 0.00 \\
\hline Spanish & 14.51 & 14.50 & 2.82 & 0.10 & 11.58 & 0.01 \\
\hline Korean & 9.00 & 8.96 & 1.65 & 0.01 & 7.30 & 0.04 \\
\hline
\end{tabular}

Table 2: 


\begin{tabular}{|c|c|c||c|c|c|c|}
\hline & tot err & segm err & del & ins & far & sub \\
\hline adapted & 43.56 & 25.44 & 5.76 & 4.82 & 14.86 & 18.12 \\
\hline baseline & 46.61 & 25.94 & 6.80 & 4.02 & 15.12 & 20.67 \\
\hline
\end{tabular}

Table 3: 


\begin{tabular}{|c|c||c|c|c|c|}
\hline tot err & segm err & del & ins & far & sub \\
\hline 38.48 & 22.33 & 6.38 & 3.83 & 12.12 & 16.15 \\
\hline
\end{tabular}

Table 4: 


\begin{tabular}{|c|c|c|c||c|c|c|c|}
\hline mode & language & tot err & segm err & del & ins & far & sub \\
\hline 1 & English & 29.87 & 28.51 & 7.63 & 0.94 & 19.94 & 1.36 \\
\hline 2 & English & 27.96 & 26.74 & 7.41 & 0.88 & 18.96 & 1.24 \\
\hline 3 & English & 28.85 & 27.45 & 6.14 & 1.01 & 20.30 & 1.40 \\
\hline 1 & Danish & 32.70 & 30.50 & 7.32 & 1.85 & 21.33 & 2.20 \\
\hline 2 & Danish & 26.47 & 25.48 & 4.15 & 2.45 & 18.88 & 0.99 \\
\hline 3 & Danish & 27.55 & 26.58 & 3.44 & 1.97 & 20.37 & 0.97 \\
\hline 1 & Italian & 23.95 & 23.95 & 6.07 & 0.02 & 17.86 & 0.00 \\
\hline 2 & Italian & 23.79 & 23.79 & 4.48 & 0.03 & 19.28 & 0.00 \\
\hline 3 & Italian & 22.89 & 22.89 & 4.03 & 0.07 & 18.79 & 0.00 \\
\hline
\end{tabular}

Table 5: 

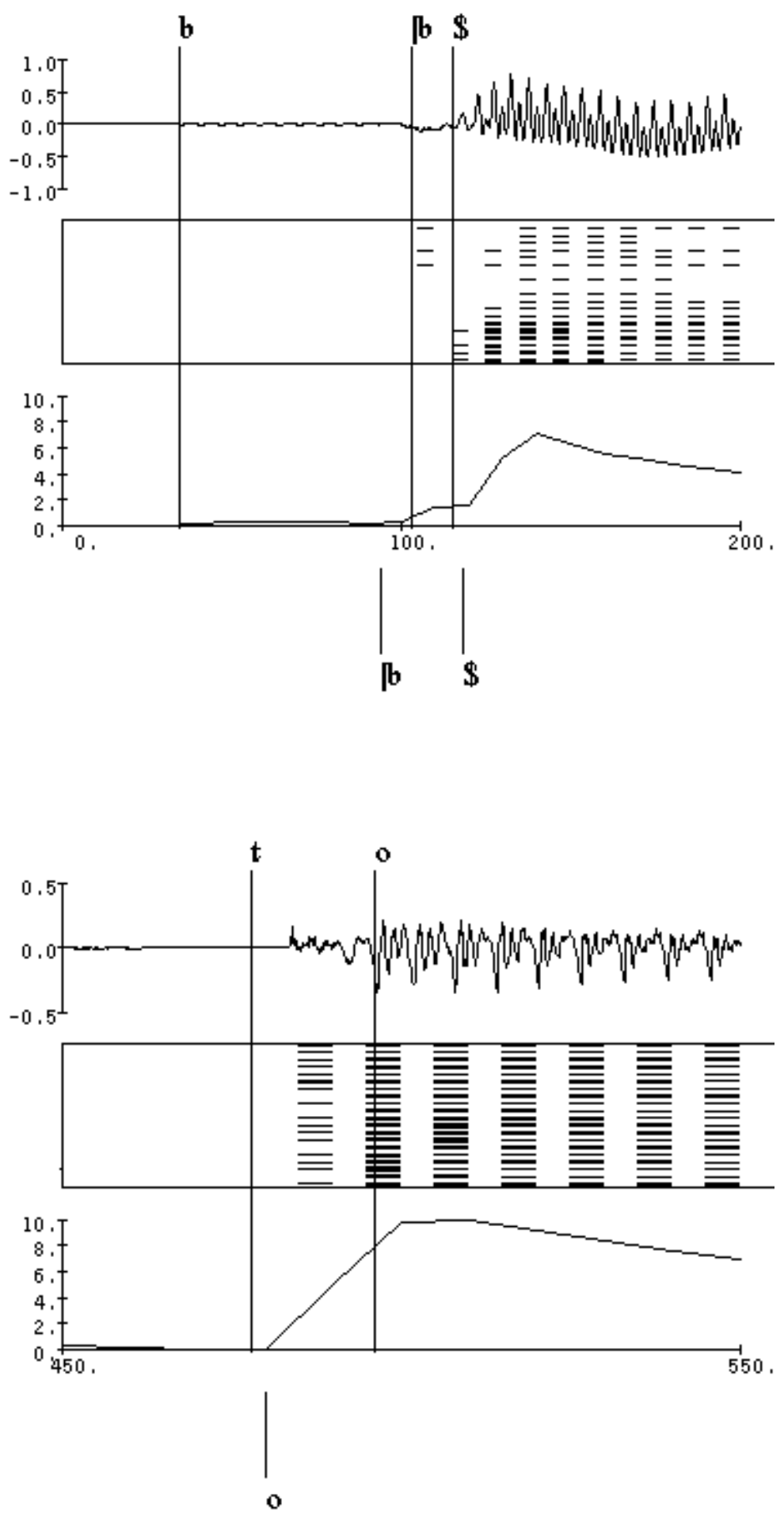

Figure 6: 

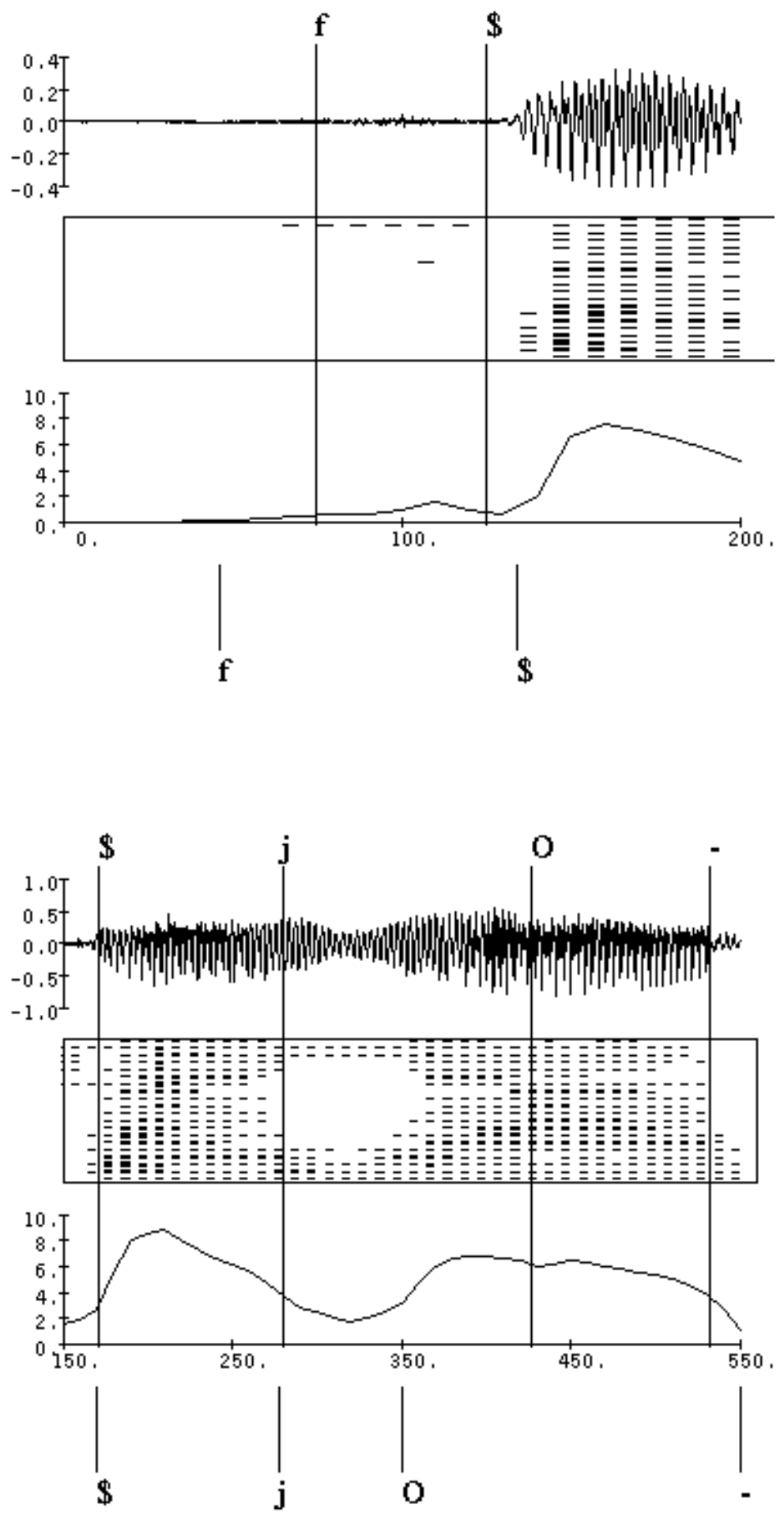

Figure 7: 

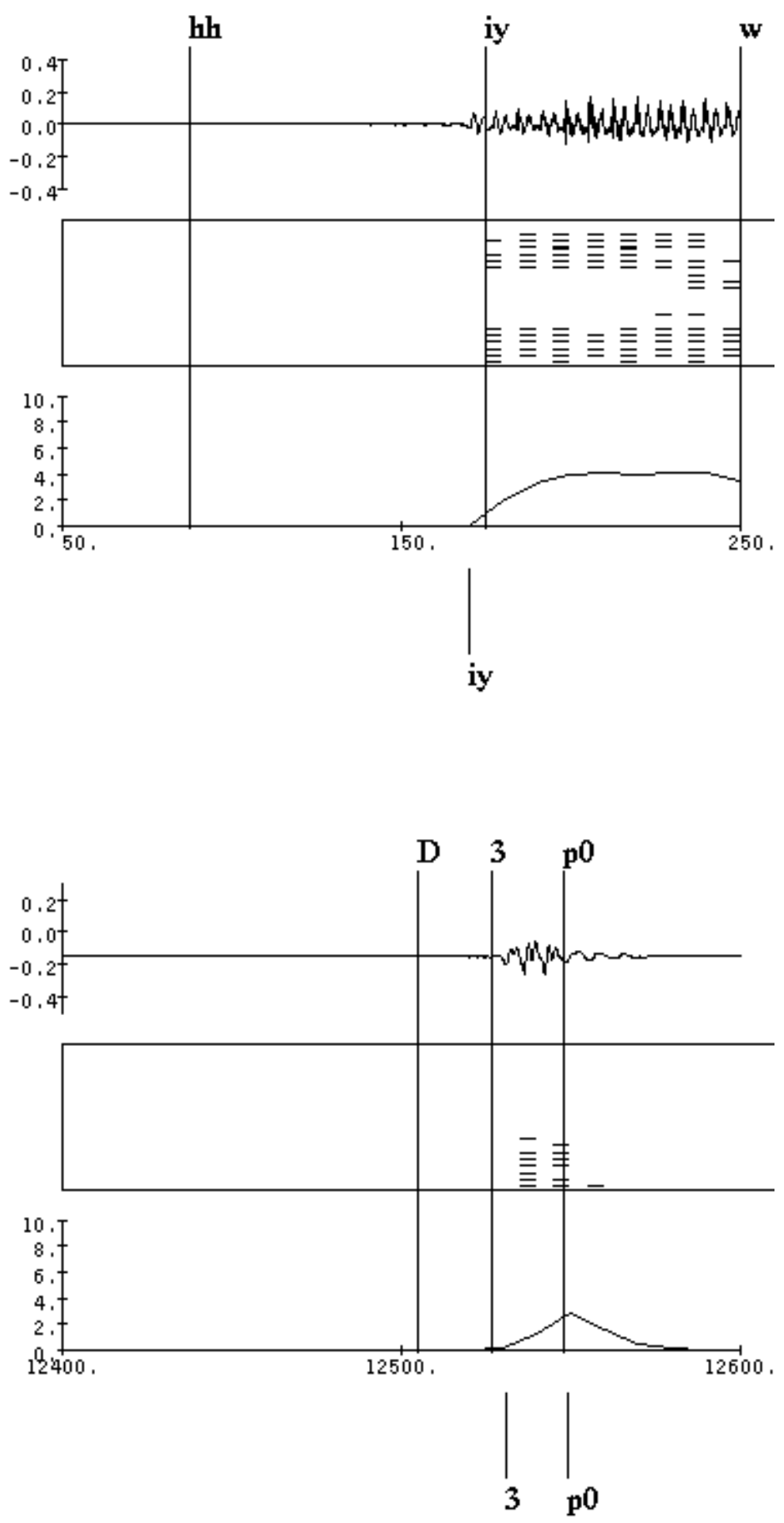

Figure 8: 

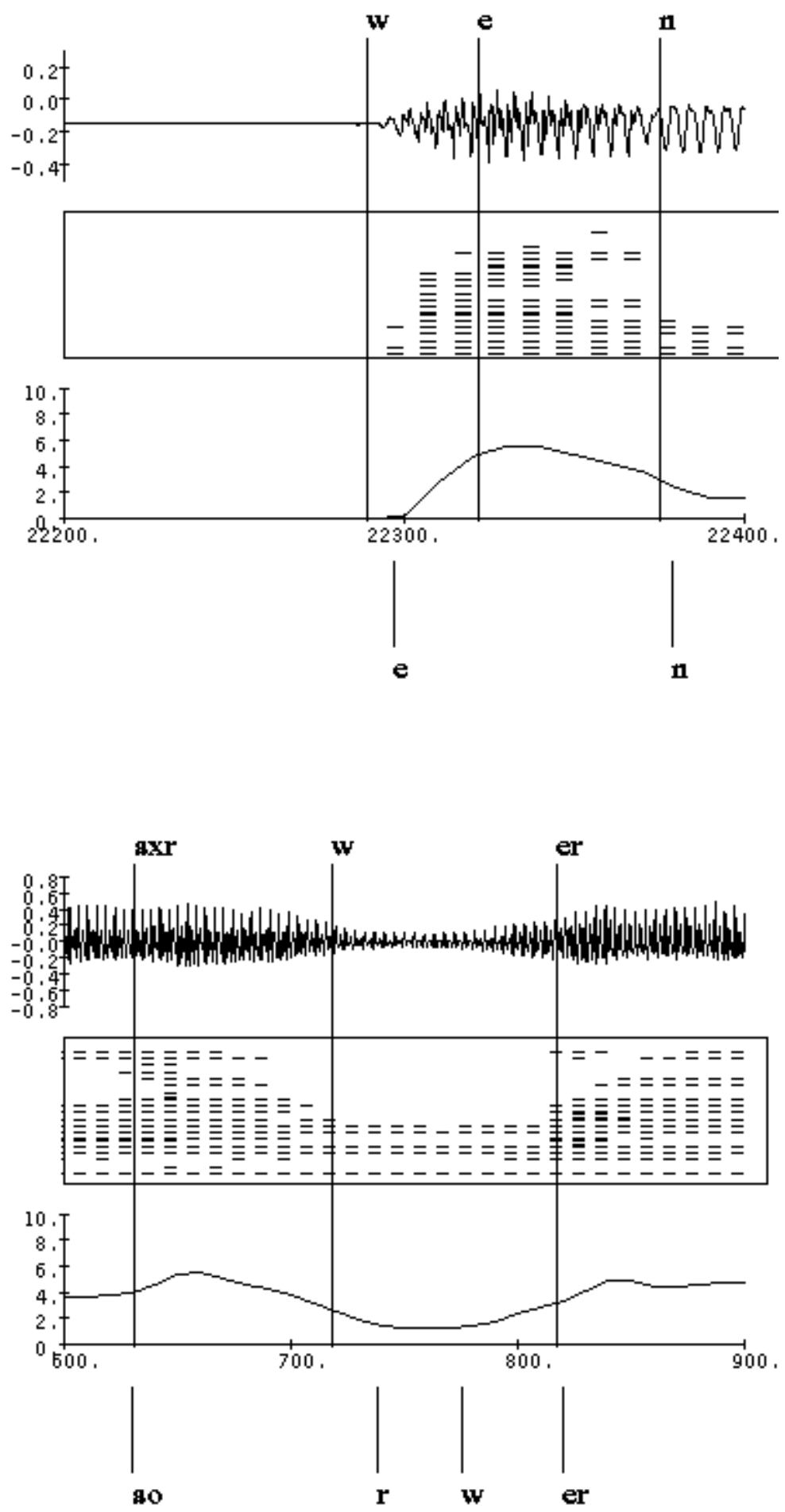

Figure 9: 

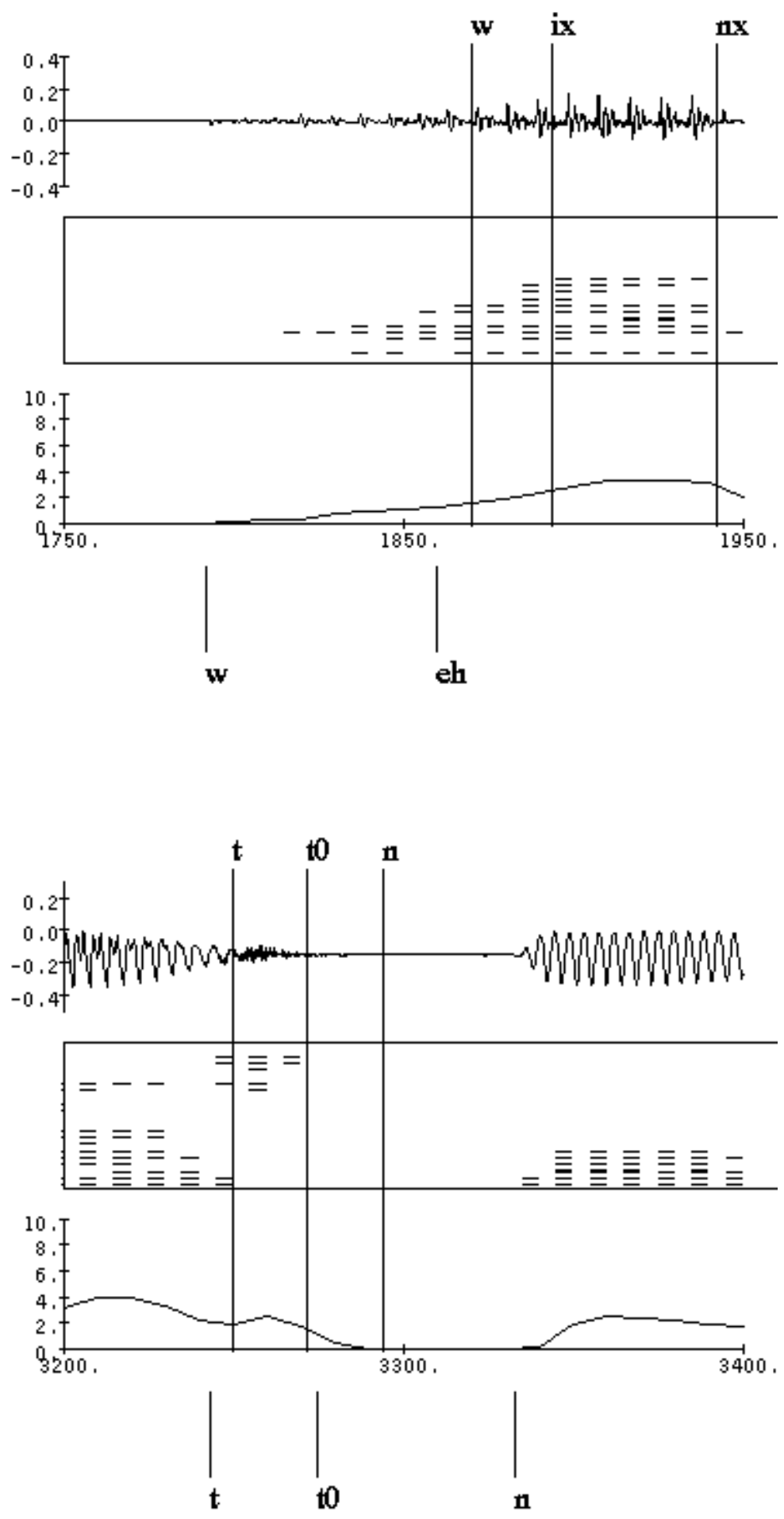

Figure 10: 